\title{
When Deep Venous Thrombosis Fails to Respond to Therapy
}

Dawn LaPorte, MS, Sharon Farber, MD, Sergei Sorin, MD, Sara Wabba, MD, Elizabeth Daneels, DO, Adam Korzenko, MS, Colin P. Kopes-Kerr, MD, JD, MPH

Background: Deep venous thrombosis in primary care is usually treated with rest, analgesics, intravenous or low-molecular-weight heparin, and coumadin. In some cases, however, a less familiar course of diagnosis and management is required.

Methods: We describe the case of a 53-year-old truck driver who had an acute deep venous thrombosis of his right lower extremity, which failed to respond to routine therapy with heparin and warfarin. A literature search was undertaken to research the differential diagnosis and management of deep venous thrombosis and to review specifically the role of venal caval filters and inherited thrombotic disorders and occult cancer in this context.

Results and Conclusion: The ultimate diagnosis in our patient appeared to be signet ring cell cancer of the colon that had metastasized to the right thigh. This case is an example of the inherent limitations of even an aggressive diagnostic and therapeutic approach to the entity of refractory deep venous thrombosis. (J Am Board Fam Pract 2003;16:246-50.)

For the usual case of acute deep venous thrombosis in primary care, all that is required for management is treatment with rest, analgesics, intravenous or low-molecular-weight heparin, and coumadin. In some cases, however, such as in younger patients without obvious risk factors or in those with recurrent disease, a less familiar course of diagnosis and management is required. We describe the following case to illustrate these issues.

\section{Methods}

We describe the case of a 53-year-old truck driver who had acute deep venous thrombosis of his right lower extremity, which failed to respond to routine therapy with heparin and warfarin. A literature search was undertaken using the key words "venous thrombosis," "recurrent," "thrombotic disorders," "occult cancer," and "vena caval filters" to research the differential diagnosis and management issues of deep venous thrombosis and specifically to review the role of venal caval filters and inherited thrombotic disorders and occult cancer in this context.

Submitted, revised, 24 December 2002.

From the Department of Family Medicine (DL, SF, SS, SW, ED, AK, CPK), State University of New York at Stony Brook. Address reprint requests to Colin P Kopes-Kerr, MD, University Hospital Department of Family Medicine, HSC L-4, 050, Stony Brook, NY 11794.

\section{Case Report}

A 53-year-old male truck driver complained to his family physician of a 3-month history of progressive pain, swelling, and firmness in his right thigh. He had been admitted to a local hospital 3 weeks earlier for painful swelling of the same thigh, and a deep venous thrombosis was confirmed by venous Doppler testing. At that time he was given heparin and coumadin. Since then, the pain in his thigh had not improved. A follow-up examination by a hematologist included testing for hereditary thrombophilias; all tests were negative. He returned to his family physician for a follow-up visit. A repeated sonogram of the right leg showed distal extension of the proximal venous thrombosis despite a therapeutic international normalized ratio (INR, 2.5). He was referred to University Hospital at Stony Brook.

The patient had a history of type 2 diabetes mellitus managed with metformin, 1,000 $\mathrm{mg}$ twice a day, and chronic hepatitis $\mathrm{C}$ secondary to intravenous drug use. He was in recovery from alcohol abuse, and he smoked 1.5 packs of cigarettes daily. He was taking famotidine, $20 \mathrm{mg}$ at bedtime, for gastroesophageal reflux disease, and paroxetine, 40 $\mathrm{mg}$ a day, for anxiety and depression. He had been married for 30 years and had no children. He had been raised solely by his grandmother and was not familiar with his family's medical history. He has 
lost between 5 and 10 pounds during the last few months.

At admission, the patient appeared to be well nourished. His blood pressure was 110/72 $\mathrm{mm} \mathrm{Hg}$, pulse 74 beats per minute, respirations $14 / \mathrm{min}$, and temperature $98.6^{\circ} \mathrm{F}$. Findings on examination of his head, ears, eyes, nose, and throat were unremarkable, and he had no adenopathy. Scattered rhonchi were heard over both lung fields. Heart sounds were normal. His abdomen was soft without masses. There was a $1.5 \times 3$-cm firm left inguinal node, and the prostate was enlarged but without nodularity. There was a $2 \times 3-\mathrm{cm}$ firm mass within the subcutaneous tissue of the posterior scrotum. The posterior part of the thigh and upper popliteal area were cool, nonerythematous, and diffusely indurated, restricting the range of motion about the knee. There was moderate tenderness over the calf.

Admitting laboratory studies disclosed the following values: a white cell count of $8,100 / \mu \mathrm{L}$ with $26 \%$ lymphocytes, $10 \%$ monocytes, and $60 \%$ neutrophils; hemoglobin was $12.2 \mathrm{~g} / \mathrm{dL}$, hematocrit $36.5 \%$ with a mean corpuscular volume of $83 / \mu \mathrm{m}^{3}$, and red cell distribution width $14 \%$; platelets $608,000 / \mu \mathrm{L}$; and the prothrombin time was 16.0 seconds, partial thromboplastin time 36.6 seconds, and INR 2.5 (on heparin and coumadin). Total bilirubin was $0.3 \mathrm{mg} / \mathrm{dL}$; alanine aminotransferase was $31 \mathrm{U} / \mathrm{L}$, aspartate aminotransferase $13 \mathrm{U} / \mathrm{L}$, alkaline phosphatase $88 \mathrm{U} / \mathrm{L}$; albumin was 3.4 $\mathrm{g} / \mathrm{dL}$, and total protein $5.9 \mathrm{~g} / \mathrm{dL}$. Results from individual clinical chemistry measurements and urinalysis were unremarkable. Erythrocyte sedimentation rate was $97 \mathrm{~mm} / \mathrm{hr}$, creatine kinase was $28 \mathrm{U} / \mathrm{L}$, and antinuclear antibodies were negative. A serum protein electrophoresis done earlier did not show any monoclonal protein or other abnormality. Findings on an electrocardiogram and chest radiography were normal.

A repeated sonogram of the right lower extremity showed thrombosis of the right common femoral vein. The patient was again given intravenous heparin. Because his thrombosis had not resolved with appropriate therapy, an inferior vena caval filter was placed.

Further evaluation with computed tomography (CT) showed minimal stranding within the right inguinal region, consistent with edema or inflammation. Magnetic resonance imaging (MRI) of the pelvis showed loss of right superficial femoral flow and extensive inflammatory process in the right thigh muscle region. There was evidence of extensive inflammation of the adductor and quadriceps musculature of the right thigh without evidence of osteomyelitis or pyomyositis.

In view of the progressive, excruciating pain and the peculiar consistency of the thigh, consultations were requested from a general surgeon, a vascular surgeon, an orthopedist, an infectious disease specialist, and an oncologist. No consultant was able to reach a specific diagnosis on initial evaluation, and a biopsy of the quadriceps muscle was recommended. The pathology report disclosed "moderate WBCs, no organisms seen. Metastatic carcinoma with focal signet ring cell features; possible primary sites include among others: upper GI, pancreas, gallbladder, biliary tree, and urinary bladder." Biopsies of the overlying skin were normal. An additional metastatic workup with an abdominal and pelvic CT scan showed thickening of the cecum. Colonoscopy showed only edematous mucosa of the rectosigmoid region. Upper endoscopy showed a normal esophagus, gastroesophageal junction, and stomach; the endoscopist, however, commented on the duodenum: "infiltration/submucosal tumor diffusely? carcinomatosis." No specific pathologic diagnosis could be made on duodenal biopsy. A sonogram of the scrotum failed to confirm the abnormality noted on physical examination. Serum testing for cancer markers showed an elevated $\beta$-human chorionic gonadotropin and CA-19-9.

The patient's condition was ultimately diagnosed as signet ring cell cancer of the colon that had metastasized to the thigh with secondary venous thrombosis of the femoral vein. The oncologists believed that the patient had a prognosis of about 3 months without therapy and 1 year with chemotherapy; the patient elected a trial of chemotherapy. He was released from the hospital shortly thereafter with follow-up visits scheduled for outpatient chemotherapy and continuing pain control. He died 6 weeks later.

\section{Discussion}

The patient initially sought care at another hospital with what appeared to be a first episode of uncomplicated right lower extremity deep vein thrombosis, which was confirmed by sonography. Potential risk factors included a history of intravenous drug abuse, but the absence of a known groin infection 
eliminated this risk factor from further consideration. ${ }^{1}$ There have also been some reports of increased risk for venous thrombosis and various abnormalities of the clotting pathway in patients with hepatitis $\mathrm{C}^{2,3}$ but in this patient hepatitis $\mathrm{C}$ did not appear to be active, as assessed by liver enzymes; it was not believed that further evaluation of this issue would influence management.

When this patient sought care a second time for symptoms of venous thrombosis, the most immediate question centered on anticoagulation therapy in a patient who already had a therapeutic INR level. Because we could find no randomized, controlled trials that addressed this question directly, we decided to intensify the anticoagulation regimen with intravenous heparin and obtained a consultant's recommendation to place an inferior vena caval filter. ${ }^{4}$ A 1998 study randomized 400 patients with proximal deep vein thrombosis who were at risk for pulmonary embolism to receive either a venal caval filter or no filter and to receive either low-molecular-weight heparin or unfractionated heparin. ${ }^{5}$ In high-risk patients with proximal deep vein thrombosis, the initial beneficial effect of vena caval filters for the prevention of pulmonary embolism was counterbalanced by an excess of recurrent deep vein thrombosis without any difference in either immediate or long-term mortality. The authors concluded: "However, because of the observed excess rate of recurrent deep-vein thrombosis and the absence of any effect on mortality among patients receiving filters, their systematic use cannot be recommended in this population." Thus in our patient we might have been too hasty when we arranged for vena caval filter placement.

After the initial management decisions were made, it became appropriate to search for other causes of recurrent or persistent venous thrombosis. The two principal predisposing factors to recurrent thrombosis are hereditary thrombotic disorders and occult cancer. In the context of recurrent deep vein thrombosis, it is important to determine what tests for inherited thrombotic disorders are indicated. The best recent answer to this question comes from The New England Fournal of Medicine. ${ }^{6}$ In a 2001 article the authors described three levels of priority for laboratory testing for patients with possible or suspected thrombotic disorders. The highest priority is testing for resistance to activated protein $\mathrm{C}$, heterozygosity or homozygosity for factor V Leiden or G20210A prothrom- bin gene mutation, homocysteine, factor VIII, and lupus anticoagulant. Intermediate priority is given to testing for decreased protein $\mathrm{C}$ activity, decreased level of free protein $\mathrm{S}$ antigen, decreased antithrombin activity, and increased titers of anticardiolipin antibodies.

In our patient these tests were done and were negative or normal. The authors described as low priority testing for dysfibrinogenemia, fibrinogen, increased factor IX activity, increased factor XI activity, and homozygous for C677T mutation in methylenetetrahydrofolate reductase gene; these tests were not done. In most patients, however, the optimal time for doing these tests is 6 months after the thrombotic event, when a decision needs to be made about continuing anticoagulant therapy. Earlier testing can be misleading because thrombosis itself can cause low antithrombin levels and elevated levels of factor VIII. Thus in our patient, if we did not find another diagnosis, we would have had to consider repeat testing.

The other general question that arises when an adult without any obvious risk factors has deep vein thrombosis is whether such an event might be related to a cancer diagnosis. A 1998 study described a cohort of patients with deep venous thrombosis or pulmonary embolism from the Danish National Registry of Patients for the years 1977 through 1992. ${ }^{7}$ The authors found a total of 15,348 patients with deep venous thrombosis and 11,305 patients with pulmonary embolism, and they observed 1,737 cases of cancer in the cohort with deep venous thrombosis compared with 1,372 expected cases. The risk was substantially elevated only during the first 6 months of follow-up and declined rapidly thereafter to a constant level slightly above 1.0 for 1 year after the thrombotic event. Forty percent of the patients with a diagnosis of cancer within 1 year after hospitalization for thromboembolism had distant metastases at the time of diagnosis.

The authors concluded that extensive cancer screening of patients with thromboembolism did not seem to be cost-effective, because such testing would have required extensive and expensive evaluations in 26,600 persons to search for the excess 304 cancers. $^{8}$ Nor was it clear that the patients' overall prognosis would have been changed. Extensive screening would also be likely to cause several other problems, including discomfort for patients and considerable psychological stress. They con- 
cluded: "Our results strongly support the pragmatic recommendation to use only simple methods of screening and to look for cancer in patients with signs and symptoms of cancer." 9

At his second admission our patient did begin to have certain signs and symptoms suggestive of cancer-localized progression of the thrombotic process despite adequate anticoagulation, unremitting pain, and specific findings localized to his thigh. These findings were variably described by different examiners but focused on the absence of redness and warmth in the skin and the presence of objective, tender swelling with distinct woodiness or hardness to the feel of the muscles of the thigh beneath the skin. The initial diagnoses considered included rheumatologic or vasculitic myositis, pyogenic myositis, and infiltrative diseases, primarily cancer. The history of weight loss during the few months preceding his admission enhanced this concern. Initial laboratory testing in the form of complete blood count, erythrocyte sedimentation rate, creatine kinase, and antinuclear antibodies was not helpful. CT scanning and MRI were able to rule in disease localized to the muscle and to rule out pyomyositis and osteomyelitis. A surgical consultation was requested to obtain a muscle biopsy from the thigh, which confirmed metastatic cancer of the "signet ring" type, presumably from a primary tumor in the gastrointestinal tract.

The term "signet ring cell carcinoma" refers histologically to a poorly differentiated adenocarcinoma with imperfectly formed glandular elements consisting of cells in which intracytoplasmic mucin compresses the nucleus against the periphery, giving the cell a characteristic signet ring appearance. Most such tumors occur in the stomach (linitis plastica), but other sites in the gastrointestinal tract, urologic tract, and breast have been reported. ${ }^{10-12}$ Elevated serum $\beta$-human chorionic gonadotropin levels have been previously reported as a tumor marker for stomach carcinomas ${ }^{13}$ and have been markers for the occurrence of bone metastasis with gastric carcinoma. ${ }^{14}$ Gastric carcinomas that are positive for $\beta$-human chorionic gonadotropin are found more frequently in poorly differentiated, advanced-stage carcinomas. Muscle metastasis as an initial manifestation of adenocarcinoma of the stomach has also been previously reported. ${ }^{15}$ Finally, although not proved in our patient, we entertained the possibility that the new nodule in the subcutaneous tissue in his scrotum was likely a metastasis; the scrotum and epididymis have been previously reported as a site of metastasis from gastric signet ring cell adenocarcinoma. ${ }^{16}$

\section{Conclusion}

This case of lower extremity deep venous thrombosis is extremely unusual. The most common and important questions family physicians face in this context are, "When is it just a simple deep venous thrombosis, and when do you have to look for other diagnoses?" Whereas most of us are ready to provide appropriate care when the diagnosis is simple deep venous thrombosis, we are not ready for the cascade of questions that arise in the few circumstances when the diagnosis is not. This case challenged us with many questions. We hope that the research we did in trying to solve the clinical problem for this patient will be useful to other family physicians who are on the front lines of the battle against deep venous thrombosis and its myriad manifestations.

\section{References}

1. Mackenzie AR, Laing RB, Douglas JG, Greaves M, Smith CC. High prevalence of iliofemoral venous thrombosis with severe groin infection among injecting drug users in North East Scotland: successful use of low molecular weight heparin with antibiotics. Postgrad Med J 2000;76:561-5.

2. Mozsik G, Figler M, Gasztonyi B, et al. [Prevalence of Leiden mutation in various gastrointestinal disorders]. Orv Hetil 2002;143:447-50.

3. Violi F, Ferro D, Basili S, et al. Increased rate of thrombin generation in hepatitis $\mathrm{C}$ virus cirrhotic patients. Relationship to venous thrombosis. J Investig Med 1995;43:550-4.

4. Luk C, Wells PS, Anderson D, Kovacs MJ. Extended outpatient therapy with low molecular weight heparin for the treatment of recurrent venous thromboembolism despite warfarin therapy. Am J Med 2001; 111:270-3.

5. Decousus H, Leizorovicz A, Parent F, et al. A clinical trial of vena caval filters in the prevention of pulmonary embolism in patients with proximal deep-vein thrombosis. Prevention du Risque d'Embolie Pulmonaire par Interruption Cave Study Group. N Engl J Med 1998;338:409-15.

6. Seligsohrn U, Lubetsky A. Genetic susceptibility to venous thrombosis. N Engl J Med 2001;344:122231.

7. Sorensen HT, Mellemkjaer L, Steffensen FH, Olsen $\mathrm{JH}$, Nielsen GL. The risk of a diagnosis of cancer after primary deep venous thrombosis or pulmonary embolism. N Engl J Med 1998;338:1169-73. 
8. Nordstrom M, Lindblad B, Anderson H, Bergqvist D, Kjellstrom T. Deep venous thrombosis and occult malignancy: an epidemiological study. BMJ 1994;308:891-4.

9. Sorensen HT, Mellemkjaer L, Steffensen FH, Olsen JH, Nielsen GL. The risk of a diagnosis of cancer after primary deep venous thrombosis or pulmonary embolism. N Engl J Med 1998;338:1169-73.

10. Nakata S, Tamura S, Morishita S, Onishi S. Depressed type primary signet ring cell carcinoma of the colon: a case report. Gastrointest Endosc 2001; 54:108-10.

11. Sarma ND, Gahukamble LN, Visweswara RH, Ramesh K, al Fituri O, Saeed SO. Primary signet ring carcinoma of the colon and rectum. Histopathology 1995;26:378-9.
12. Almagro UA. Primary signet-ring carcinoma of the colon. Cancer 1983;52:1453-7.

13. Rau B, Below C, Haensch $W$, Liebrich $W$, von Schilling C, Schlag PM. [Significance of serum betahCG as a tumor marker for stomach carcinoma.] Langenbecks Arch Chir 1995;380:359-64.

14. Yonemura Y, Oyama S, Sugiyama K, et al. Human chorionic gonadotropin in gastric carcinoma. A useful marker for bone metastasis. Int Surg 1989;74: 84-7.

15. Pestalozzi BC, von Hochstetter AR. [Muscle metastasis as initial manifestation of adenocarcinoma of the stomach], Schweiz Med Wochenschr 1998;128: 1414-7.

16. Ozdal OL, Yakupoglu YK, Cicek A, Erdem O, Memis L, Memis A. Epididymal metastasis from gastric signet ring cell adenocarcinoma. Can J Urol 2002;9: 1498-9. 